\title{
An Improved Caching Strategy for Training SVMs
}

\author{
Liang Zhou $^{1}$ Fen Xia $^{1}$ Yanwu Yang ${ }^{1}$ \\ ${ }^{1}$ Institute of Automation,Chinese Academy of Sciences, Beijing 100080, P. R. China
}

\begin{abstract}
Computational complexity is one of the most important issues while dealing with the training of Support Vector Machines(SVMs), which is done by solving corresponding linear constrained convex quadratic programming problems. The stateof-the-art training of SVMs takes iterative decomposition strategies that focus on working-set selection to solve quadratic programming problems. Shrinking and caching are two indispensable strategies to reduce the complexity of the decomposition process. Yet, most existing caching strategies mainly consider usage records of samples, while ignoring probabilities of samples being selected into working sets. These probabilities might determine the efficiency of caching. This paper proposes an improved caching strategy by taking into account these probabilities of samples being selected into working sets, to reduce computational costs of kernel evaluations in the training of SVMs. Experiments on several benchmark data sets show that our caching strategy is more efficient than those existing ones.
\end{abstract}

Keywords: Support vector machine, Working set selection, Shrinking, Caching, Kernel evaluation

\section{Introduction}

Support Vector Machines(SVMs) are a family of powerful statistical learning techniques for pattern recognition, regression and density estimation problems. SVMs have proven to be effective in many practical applications. SVMs learning methods are based on the structural risk minimization (SRM) induction principle, which is derived from the statistical learning theory [1]-[3]. The problem of training SVMs is converted to a linear constrained convex quadratic programming problem.

A lot of commercial packages are used to solve quadratic programming problems. In these packages, a whole kernel matrix $Q$ is held in the memory, thus the quadratic programming problem has the space complexity $O\left(l^{2}\right)$ and the time complexity $O\left(l^{3}\right)$, where $l$ is the size of training set. When $l$ is too large, it's impossible to afford the space and the time. Decomposition algorithms are mainstream approaches to tackle this problem. They decompose a large-scale optimization problem into several small-scale sub-problems. By solving a series of small-scale sub-problems sequently, they obtain a solution to the large-scale optimization problem. At each step a fixed-size subset(called working-set B) of the whole training set is chosen to form a small-scale optimization sub-problem. When the small-scale optimization sub-problem is solved, some samples of the working-set are substituted by those outside (of working set) with some working-set selection strategies. Then the problem of solving the small-scale sub-problem is formulated in the same fashion through an iterative process. Finally, decomposition algorithms terminate when some criteria (e.g. KKT conditions for all samples) are satisfied.

Kernel evaluations are the main computational complexity. So the number of kernel evaluations is a good criteria to evaluate the performance of training SVMs. Some algorithms are proposed to reduce the total number of iteration [4] or to minimize the number of kernel evaluations per iteration $[5]$.

The state-of-the-art training of SVMs takes iterative decomposition strategies that focus on working-set selection to solve quadratic programming problems. A series of kernel evaluations are repeated in the training of SVMs, therefore, caching strategies can be used to avoid the recomputation of those evaluations. This implies searching for an elegant trade-off between memory consumption and the training time. In many tasks the number of Support Vectors $(\mathrm{SVs})^{1}$ is much smaller than the number of training samples. This implies that, kernel evaluations can be dealt by only considering evaluations relevant to those SVs, which approximates to some shrinking strategies.

\footnotetext{
${ }^{1}$ In (2), support vectors refers to samples whose corresponding $\alpha_{i}$ is not zero.
} 
Shrinking and caching are two indispensable strategies to reduce the complexity of the decomposition process. Joachims [6] first took shrinking and caching strategies to train SVMs, and previous experiments demonstrated that shrinking and caching had very good effects. To the best of our knowledge, there are two popular packages of training SVMs using caching strategies: $S V M^{\text {light }}$ and $L I B S V M$. The former records the times of (samples) being selected into the working-set, then replaces samples with least times in caching by those being selected. The latter views the caching as a queue. and deletes the head of the queue while adding samples being selected into the tail of the queue. Most existing caching strategies mainly consider usage records of samples, while ignoring probabilities of samples being selected into working sets. These probabilities might determine the efficiency of caching. In this paper, we propose an improved caching strategy with consideration of these probabilities to save computational costs of kernel evaluation in the training of SVMs. Experiments on several benchmark data sets show that our caching strategy is more efficient than those existing ones.

The remaining of the paper is organized as follows. Section 2 introduces the standard SVMs. Section 3 discusses the general decomposition algorithm and some existing working-set selection algorithms. Section 4 describes most existing shrinking and caching strategies. An improved caching strategy is proposed in Section 5. Experiments and discussions are presented in Section 6 . Section 7 concludes the paper.

\section{Standard support vector machines}

To facilitate the reading, here are some important notations in this paper:

- $l$ : size of training samples

- $n$ : dimension of the sample's feature

- $\phi(x)$ : the mapping function

- $\mathbf{w}$ : weights vector

- $\alpha$ : Lagrange multipliers vector

- $b$ : bias term

- $K$ : sample inner product $=<\phi\left(x_{i}\right), \phi\left(x_{j}\right)>$

- $\epsilon$ : stop condition

- $Q$ : kernel matrix $=y_{i} y_{j}<\phi\left(x_{i}\right), \phi\left(x_{j}\right)>$

- $B$ : working set

- e: the vector of all ones

- C: regularization parameter $(\geq 0)$

- $M(\alpha): \min _{i \in I_{\text {low }}(\alpha)}-y_{i} \nabla f(\alpha)_{i}$
- $m(\alpha): \max _{i \in I_{u p}(\alpha)}-y_{i} \nabla f(\alpha)_{i}$

- $I_{u p}(\alpha):\left\{t \mid \alpha_{t} \leq C, y_{t}=1 \| \alpha_{t} \geq 0, y_{t}=\right.$ $-1\}$

- $I_{\text {low }}(\alpha):\left\{t \mid \alpha_{t} \leq C, y_{t}=-1 \| \alpha_{t} \geq 0, y_{t}=\right.$ $1\}$,

In this paper, we just consider standard SVMs(1norm, proposed by V.N.Vapnik[1][2] for classification). Given training vectors $\mathbf{x}_{i} \in R^{n}, i=1, \ldots, l$, in two classes, and a vector $\mathbf{y} \in R^{l}$ such that $y_{i} \in\{1,-1\}$, the standard SVMs solve the following primal problem:

$$
\begin{array}{rc}
\min _{\mathbf{w}, b, \xi} & \frac{1}{2} \mathbf{w}^{T} \mathbf{w}+C \sum_{i=1}^{l} \xi_{i} \\
\text { subject to } & y_{i}\left(\mathbf{w}^{T} \phi\left(\mathbf{x}_{i}\right)+b \geq 1-\xi_{i},\right. \\
& \xi_{i} \geq 0, i=1, \ldots, l .
\end{array}
$$

Its dual problem is

$$
\begin{array}{rc}
\min _{\alpha} & \frac{1}{2} \alpha^{T} Q \alpha-\mathbf{e}^{T} \alpha \\
\text { subject to } & 0 \leq \alpha_{i} \leq C, i=1, \ldots, l, \\
& \sum_{i=1}^{l} y_{i} \alpha_{i}=0 .
\end{array}
$$

Usually, we solve the dual problem (2), as it is easier to solve (2) than (1).

\section{Decomposition algorithm and working set selection}

In this section, we introduce the history and details of decomposition algorithm. First, the simplest heuristic is known as chunking by V.N.Vapnik[1]. And then, Osuna [7] proved that if $\mathrm{B}$ contains a variable, which violates the optimality condition, the object function will have a strict improvement when the sub-optimal is re-optimized. Osuna also gave an improved algorithm for training SVMs.

The Sequential Minimal Optimization (SMO) [8] algorithm is derived by taking the idea of the decomposition method to its extreme and optimizing a minimal subset of just two points ${ }^{2}$ at each iteration. The power of this technique resides in the fact that the optimization problem for two data points admits an analytical solution, eliminating the need to use an iterative quadratic programme optimizer as part of the algorithm. In general a decomposition algorithm can be formulated as table 1 .

\footnotetext{
${ }^{2}$ The requirement that the condition $\sum_{i=1}^{l} y_{i} \alpha_{i}=0$ is enforced throughout the iterations implies that the smallest number of $\mathrm{B}$ is 2 .
} 


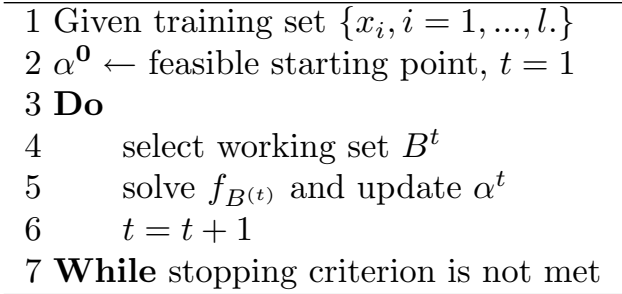

Table 1: Decomposition Algorithm In General

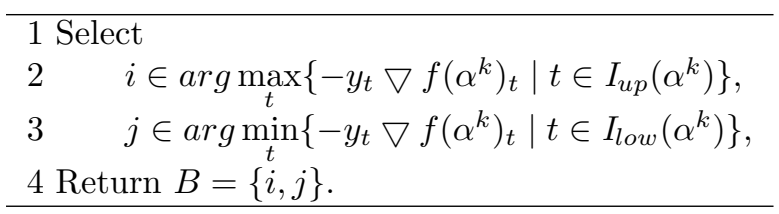

Table 2: Maximal Violating Pair

The fourth step in table 1 decides the process of the algorithm iterative, and the convergence of the decomposition algorithm depends strongly on the working set selection algorithm. There are two main categories of working set selection algorithm, one uses first-order information and the other uses second-order information. The first-order information algorithm selects pairs of variables that mostly violate(MVP) $[9]^{3}$, which is formulated as table 2 , the Karush-Kuhn-Tucker(KKT) condition for optimality.

So far, there are two algorithms taking secondorder information into account(LIBSVM-2.8 [4] [10] and HMG [5]). Although both of them use secondorder information, ultimately they could not avoid all $C_{l}^{2}$ pairs to get the optimal B. In fact, they all use the greedy strategy. LIBSVM-2.8 selects the same $i$ as MVP, and then checks only $O(l)$ pairs to decide $j$, while HMG takes one variable of the previous $B^{t}$ into the current B. They both have disadvantages in per iteration: LIBSVM-2.8 can not guarantee that the corresponding kernels ${ }^{4}$ of $i$ are available in the matrix cache, while HMG can only take full advantage of a sample point of information in each iteration as it takes one point of the previous $B^{t}$. The LIBSVM-2.8 and HMG working set selection algorithm are presented in table 3 and table 4 respectively. Next section will give the details of shrinking and caching strategies used in popular software (LIBSVM and $\left.S V M^{\text {light }}\right)$ of training SVMs.

\footnotetext{
${ }^{3}$ Detailed derivation is specified in [4] [9].

${ }^{4}$ Evaluating the sub-object function should use the kernel $Q_{i j}, j=1, \ldots, l$.
}

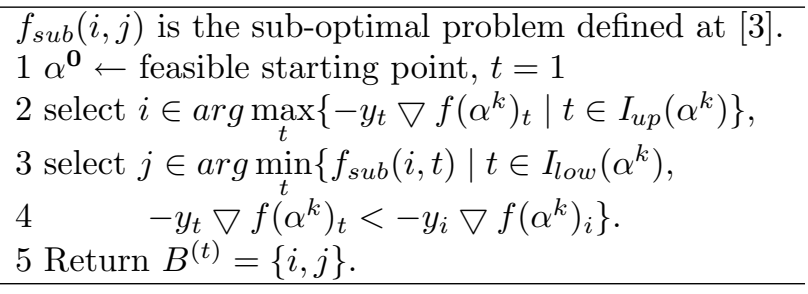

Table 3: LIBSVM-2.8

\section{Shrinking and caching}

Shrinking and Caching are two very effective strategies for training SVMs. They are first proposed by Joachims [6], and implemented in software $S V M^{\text {light }}$. Shrinking is based on the idea that those samples, whose $\alpha_{i}$ are in the value of the border, may not be changed in the future training. So those samples can be shrunken in the training procedure, then the whole training problem becomes smaller and easier to be solved. P.-H. Chen [11] proves the following theorem:

Theorem 1 Assume Q is positive semi-definite and the decomposition algorithm uses LIBSVM2.8 working set selection algorithm. Let $I \equiv\{i \mid$ $-y_{i} \nabla f(\alpha)_{i}>M(\alpha)$ or $\left.-y_{i} \nabla f(\alpha)_{i}<m(\alpha)\right\}$. There is $\bar{k}$ such that after $k>\bar{k}$, every $\alpha_{i}^{k}, i \in I$ has reached the unique and bounded optimal solution. It remains the same in all subsequent iterations and $i \in I$ is not in the following set:

$$
\left\{t \mid M\left(\alpha^{k}\right) \leq-y_{t} \nabla f\left(\alpha^{k}\right)_{t} \leq m\left(\alpha^{k}\right)\right\} .
$$

The software $L I B S V M^{5}$ shrinks the $\alpha_{i}$ considering set (3), while $S V M^{\text {light }}$ is based on the idea that any $\alpha_{i}$ which has stayed at the same bound for a certain number of iterations can be removed.

Caching strategy can effectively improve the efficiency of procedures and save training time. However, in large-scale data processing, as lack of memory space, caching strategy has to be used. There are two different relatively simple caching strategies in existing software. The software $L I B S V M$ implements a simple least-recent strategy for caching. It dynamically caches only recently used kernel of $Q_{B^{t}}$. Its details are shown in table 5 .

And the idea of $S V M^{\text {light }}$, which is based on the least-used strategy, is similar with $L I B S V M$ 's least-recent strategy. Therefore, its strategy is to preferentially delete the $\alpha_{i}$, which is the least num-

\footnotetext{
${ }^{5}$ As the implement of HMG is updated from software $L I B S V M$, it uses the same shrinking and caching techniques as $L I B S V M$.
} 


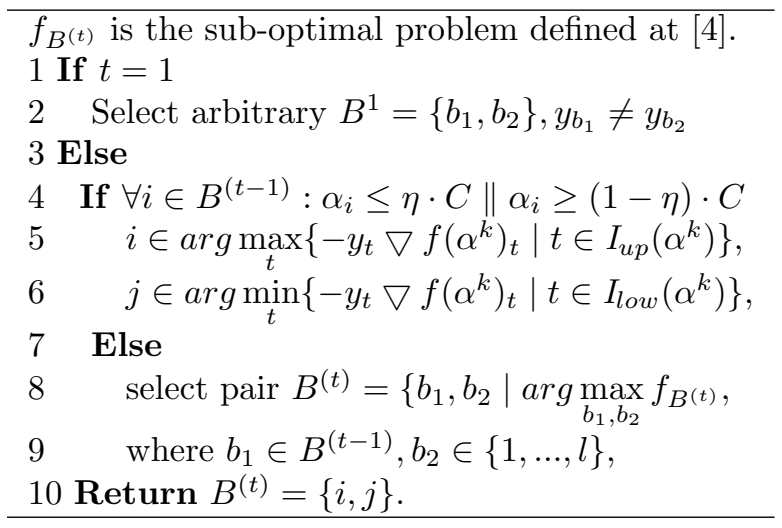

Table 4: Hybrid Maximum-Gain $0<\eta \ll 1$

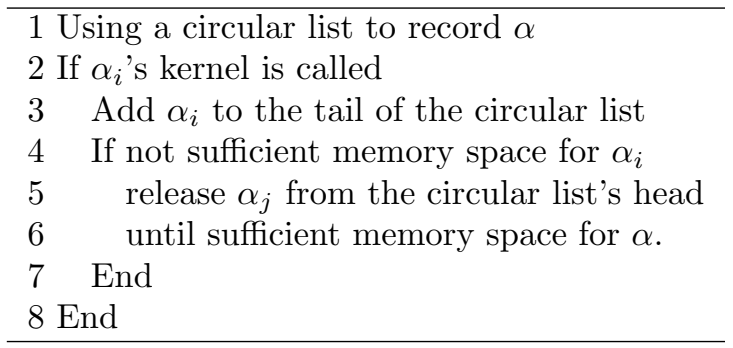

Table 5: Caching in $L I B S V M$

ber of kernel used, in the current cache. Also, details are shown in table 6 .

\section{An improved caching strat- egy}

Currently, the focus of decomposition algorithm is how to select the working set. The most popular algorithm is using the second-order information of the sub-optimal problem to select working

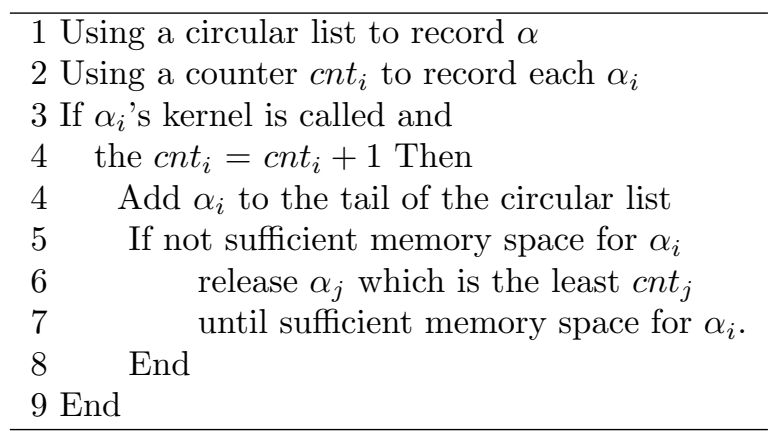

Table 6: Caching in $S V M^{\text {light }}$

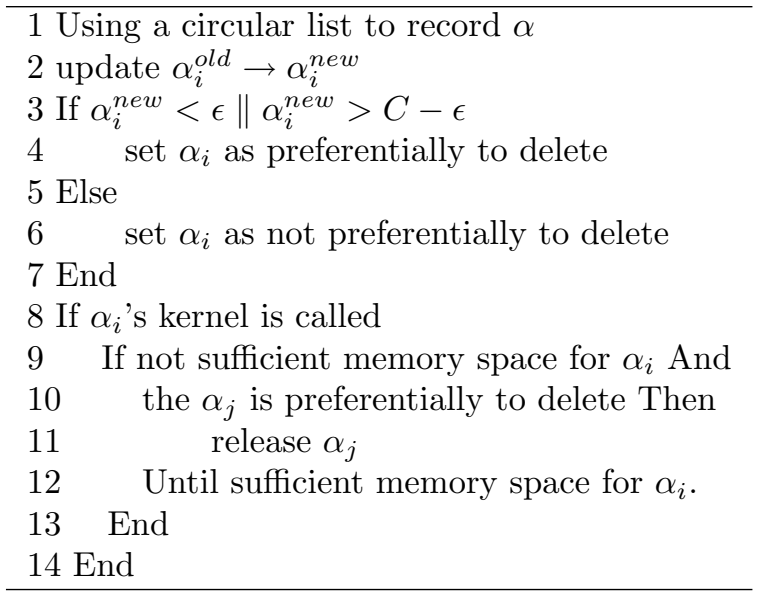

Table 7: A New Caching Strategy

set. Just as mentioned in section 3 , there are two main working set selection algorithms using secondorder information: HMG and LIBSVM-2.8. The difference between LIBSVM-2.8 and HMG working set selection algorithm, which are specified in table 3 and 4 respectively, is that they use different way to solve the similar second-order suboptimal problem. Although the working set selection algorithm decides the training process, different strategies of shrinking and caching will have different effect on saving time. As far as shrinking and caching are concerned, the implement of HMG is the same as LIBSVM-2.8. Compared to $L I B S V M, S V M^{\text {light }}$ uses first-order information of the sub-optimal problem to select working set, as well as different shrinking and caching strategies. This paper focuses on proposing a generic caching strategy that is based on the combination of working set selection algorithm and shrinking strategy. The specific strategy can be referred to table 7 .

This strategy fully takes into account the interdependency of the working set selection, shrinking and caching, which are three different main aspects in the special optimization problem of SVMs. As in the HMG working set selection algorithm, if current $\alpha_{i} \in B$ is in the border, this $\alpha_{i}$ will have a great probability of not being re-selected. Meanwhile in the $L I B S V M$, the nature of shrinking algorithm is also removing the border $\alpha_{i}$ from the training set. As we already know the fact that some samples will no longer be selected into set $B$, we can base on this real-time information to improve caching strategy. As in the table 7, if $\alpha_{i}^{\text {new }}<\epsilon \| \alpha_{i}^{\text {new }}>C-\epsilon$, the $\alpha_{i}^{\text {new }}$ will have a great probability of not being re-selected to set $B$. Based on the above considerations, we present this generic caching strategy, 


\begin{tabular}{|l|l|l|l|}
\hline Data set & \# data & \# feature & \# class \\
\hline a9a & 32,561 & 123 & 2 \\
ijcnn 1 & 49,990 & 22 & 2 \\
w8a & 49,749 & 300 & 2 \\
protein & 14,895 & 357 & 3 \\
usps & 7,291 & 256 & 10 \\
\hline
\end{tabular}

Table 8: Data Statistics

which is preferentially to delete the $\alpha_{i}$ on the border. This strategy is not ever considered in early implement. Now, we propose the kernel evaluation rate for evaluation criteria :

$$
\text { Rate }_{k e}=\frac{\# \text { total kernel evaluations }}{\# \text { total kernel calls }}
$$

Total kernel evaluations refers to the actual amount of computation kernel, while total kernel calls refers to the actual demand of kernel. Clearly, caching will not affect the iteration of training process, the entire iterative process is decided by the working set selection algorithms. But as a caching strategy, a lower kernel evaluation rate means that more kernel evaluations are avoided. This can be calculated from the total amount of kernel evaluation, a common evaluation criteria, to be tested.

\section{Experiments}

In this section we use $L I B S V M$ software as our benchmark. We will adapt the caching strategy of the primitive $L I B S V M$ to Bound (we proposed) and $U$ sed(caching strategy in software $S V M^{\text {light }}$ ). we name primitive $L I B S V M$ 's caching strategy as Recent. With such three caching strategies implement, comparison will be performed between these three versions.

Five data $\operatorname{sets}(a 9 a, i j c n n 1, w 8 a$, protein and $u s p s)^{6}$ are considered and we only take classification for comparison. We compare the performance of our caching strategy against the existing ones. Data statistics are specified in table 8.

\subsection{Experimental settings}

We know that the SVMs can be used for classification, one class, regression and density estimation. We use classification, the basic usage of SVMs, to

\footnotetext{
${ }^{6}$ All data sets presented in this paper are available at http://www.csie.ntu.edu.tw/ cjlin/libsvmtools/datasets/.
}

evaluate different caching strategies. In classification, different SVMs parameters such as C in (1) and kernel parameters affect the training procedure. It is difficult to evaluate the three caching strategies under every parameter setting. Here we use the similar experiments setting as [4]. To have a fair comparison, we simulate how one uses SVMs in practice. We consider two following training procedures:

1. "Parameters selection": using five-fold cross validation to find the best parameters.

2. "Final training": train the whole set.

In "Parameters selection" step, we use a fixed small caching space to evaluate the three caching strategies, while in "final training" step, we use various caching space. Each step will be with or without shrinking. For simpleness, we only use the RBF $\operatorname{kernel}(\gamma=1$ for this experiments):

$$
K\left(x_{i}, x_{j}\right)=e^{-\gamma\left\|x_{i}-x_{j}\right\|^{2}}
$$

For completeness we give the details of experiment platform here. The experiments are reported to have been carried out on a $2.4 \mathrm{GHz}$ Pentium 4 processor with $512 \mathrm{M}$ of RAM running cygwin, using $\mathrm{g}++3.4 .4$ compiler.

\subsection{Comparison and analysis}

We train SVMs on all data sets as shown in Table 8 to compare these three caching strategies. The kernel evaluation rate(4) is used as the evaluation criteria of performance. Results are shown in table 9 and table 10. Table 9 shows results of "Parameters selection" procedure, which uses fixed caching space $(10 \mathrm{Mb})$ and different $\mathrm{C}$ varying among 1,10 and 100. Table 10 is for the "Final training" procedure. It uses the same $\mathrm{C}(100)$, but different caching space(from 10 to 200). In the table 9 and table 10, the lowest values are boldfaced, which mean more kernel evaluations are saved.

As the number of kernel evaluation is independent of the caching space, the training time is proportional to kernel evaluation rate. In the "Final training" procedure, the processes of training with the three caching strategies are same, which means the total numbers of kernel evaluation are also same, while the caching spaces are different. Table 10 shows that the Bound caching strategy performs best and the $U$ sed is the worst among the three caching strategies. In our version of $U$ sed, if the sample is deleted from the circular list, the $c_{n} t_{i}$ will be set 0 . This might be not appropriate. We leave it for the further work. 


\begin{tabular}{|c|c|c|c|}
\hline \multirow[b]{2}{*}{ strategy } & \multicolumn{3}{|c|}{ a9a,No shrinking } \\
\hline & 1 & 10 & 100 \\
\hline Recent & 0.374 & 0.412 & 0.534 \\
\hline Used & 0.412 & 0.547 & 0.647 \\
\hline \multirow[t]{3}{*}{ Bound } & 0.368 & 0.402 & 0.532 \\
\hline & \multicolumn{3}{|c|}{ a9a, shrinking } \\
\hline & 1 & 10 & 100 \\
\hline Recent & 0.418 & 0.429 & 0.412 \\
\hline Used & 0.425 & 0.459 & 0.584 \\
\hline \multirow[t]{3}{*}{ Bound } & 0.420 & 0.427 & 0.410 \\
\hline & \multicolumn{3}{|c|}{ ijcnn1,No shrinking } \\
\hline & 1 & 10 & 100 \\
\hline Recent & 0.384 & 0.339 & 0.311 \\
\hline Used & 0.404 & 0.429 & 0.586 \\
\hline \multirow[t]{3}{*}{ Bound } & 0.379 & 0.327 & 0.303 \\
\hline & \multicolumn{3}{|c|}{ ijcnn1, shrinking } \\
\hline & 1 & 10 & 100 \\
\hline Recent & 0.408 & 0.411 & 0.424 \\
\hline Used & 0.412 & 0.464 & 0.490 \\
\hline \multirow[t]{3}{*}{ Bound } & 0.408 & 0.411 & 0.421 \\
\hline & \multicolumn{3}{|c|}{ w8a,No shrinking } \\
\hline & 1 & 10 & 100 \\
\hline Recent & 0.410 & 0.457 & 0.493 \\
\hline Used & 0.453 & 0.559 & 0.632 \\
\hline \multirow[t]{3}{*}{ Bound } & 0.395 & 0.443 & 0.491 \\
\hline & \multicolumn{3}{|c|}{ w8a, shrinking } \\
\hline & 1 & 10 & 100 \\
\hline Recent & 0.435 & 0.433 & 0.341 \\
\hline Used & 0.457 & 0.540 & 0.570 \\
\hline \multirow[t]{3}{*}{ Bound } & 0.433 & 0.422 & 0.336 \\
\hline & \multicolumn{3}{|c|}{ protein,No shrinking } \\
\hline & 1 & 10 & 100 \\
\hline Recent & 0.387 & 0.242 & 0.303 \\
\hline Used & 0.402 & 0.606 & 0.468 \\
\hline \multirow[t]{3}{*}{ Bound } & 0.387 & 0.230 & 0.290 \\
\hline & \multicolumn{3}{|c|}{ protein, shrinking } \\
\hline & 1 & 10 & 100 \\
\hline Recent & 0.412 & 0.380 & 0.397 \\
\hline Used & 0.428 & 0.446 & 0.594 \\
\hline \multirow[t]{3}{*}{ Bound } & 0.416 & 0.378 & 0.390 \\
\hline & \multicolumn{3}{|c|}{ usps,No shrinking } \\
\hline & 1 & 10 & 100 \\
\hline Recent & 0.212 & 0.069 & 0.053 \\
\hline Used & 0.212 & 0.069 & 0.053 \\
\hline \multirow{3}{*}{ Bound } & 0.212 & 0.069 & 0.052 \\
\hline & \multicolumn{3}{|c|}{ usps, shrinking } \\
\hline & 1 & 10 & 100 \\
\hline Recent & 0.212 & 0.069 & 0.054 \\
\hline Used & 0.212 & 0.069 & 0.054 \\
\hline Bound & 0.212 & 0.069 & 0.054 \\
\hline
\end{tabular}

Table 9: caching space $=10 \mathrm{Mb}, \mathrm{cv}=5$ fold, $\mathrm{C}=$ ?

\begin{tabular}{|c|c|c|c|c|c|}
\hline \multirow{2}{*}{ strategy } & \multicolumn{5}{|c|}{ a9a,No shrinking } \\
\hline & 10 & 20 & 40 & 100 & 200 \\
\hline Recent & 0.569 & 0.501 & 0.375 & 0.105 & 0.047 \\
\hline Used & 0.645 & 0.642 & 0.610 & 0.590 & 0.505 \\
\hline \multirow[t]{3}{*}{ Bound } & 0.568 & 0.499 & 0.370 & 0.094 & 0.038 \\
\hline & \multicolumn{5}{|c|}{ a9a, shrinking } \\
\hline & 10 & 20 & 40 & 100 & 200 \\
\hline Recent & 0.433 & 0.393 & 0.349 & 0.282 & 0.218 \\
\hline Used & 0.593 & 0.577 & 0.556 & 0.524 & 0.485 \\
\hline \multirow[t]{3}{*}{ Bound } & 0.431 & 0.391 & 0.345 & 0.276 & 0.214 \\
\hline & \multicolumn{5}{|c|}{ ijcnn1,No shrinking } \\
\hline & 10 & 20 & 40 & 100 & 200 \\
\hline Recent & 0.373 & 0.244 & 0.150 & 0.115 & 0.105 \\
\hline Used & 0.573 & 0.572 & 0.563 & 0.326 & 0.287 \\
\hline \multirow[t]{3}{*}{ Bound } & 0.365 & 0.230 & 0.125 & 0.099 & 0.099 \\
\hline & \multicolumn{5}{|c|}{ ijcnn1, shrinking } \\
\hline & 10 & 20 & 40 & 100 & 200 \\
\hline Recent & 0.436 & 0.410 & 0.375 & 0.337 & 0.318 \\
\hline Used & 0.493 & 0.488 & 0.478 & 0.463 & 0.457 \\
\hline \multirow[t]{3}{*}{ Bound } & 0.435 & 0.407 & 0.370 & 0.335 & 0.317 \\
\hline & \multicolumn{5}{|c|}{ w8a,No shrinking } \\
\hline & \begin{tabular}{l|l}
10 \\
\end{tabular} & \begin{tabular}{|l|}
20 \\
\end{tabular} & 40 & 100 & 200 \\
\hline Recent & 0.523 & 0.433 & 0.273 & 0.053 & 0.032 \\
\hline Used & 0.636 & 0.621 & 0.534 & 0.479 & 0.349 \\
\hline \multirow[t]{3}{*}{ Bound } & 0.521 & 0.429 & 0.265 & 0.042 & 0.028 \\
\hline & \multicolumn{5}{|c|}{ w8a, shrinking } \\
\hline & 10 & 20 & 40 & 100 & 200 \\
\hline Recent & 0.400 & 0.267 & 0.194 & 0.136 & 0.100 \\
\hline Used & 0.570 & 0.544 & 0.517 & 0.483 & 0.298 \\
\hline \multirow[t]{3}{*}{ Bound } & 0.396 & 0.260 & 0.187 & 0.126 & 0.088 \\
\hline & \multicolumn{5}{|c|}{ protein,No shrinking } \\
\hline & \begin{tabular}{|l|l|}
10 \\
\end{tabular} & \begin{tabular}{l|l}
20 \\
\end{tabular} & 40 & 100 & 200 \\
\hline Recent & 0.412 & 0.191 & 0.110 & 0.078 & 0.060 \\
\hline Used & 0.611 & 0.604 & 0.590 & 0.507 & 0.274 \\
\hline \multirow[t]{3}{*}{ Bound } & 0.399 & 0.168 & 0.087 & 0.061 & 0.052 \\
\hline & \multicolumn{5}{|c|}{ protein, shrinking } \\
\hline & 10 & 20 & 40 & 100 & 200 \\
\hline Recent & 0.432 & 0.369 & 0.299 & 0.224 & 0.172 \\
\hline Used & 0.594 & 0.587 & 0.565 & 0.440 & 0.244 \\
\hline \multirow[t]{3}{*}{ Bound } & 0.425 & 0.358 & 0.280 & 0.187 & 0.153 \\
\hline & \multicolumn{5}{|c|}{ usps,No shrinking } \\
\hline & \begin{tabular}{l|l}
10 \\
\end{tabular} & \begin{tabular}{l|l}
20 \\
\end{tabular} & 40 & 100 & 200 \\
\hline Recent & 0.052 & 0.052 & 0.052 & 0.052 & 0.052 \\
\hline Used & 0.052 & 0.052 & 0.052 & 0.052 & 0.052 \\
\hline \multirow[t]{3}{*}{ Bound } & 0.052 & 0.052 & 0.052 & 0.052 & 0.052 \\
\hline & \multicolumn{5}{|c|}{ usps, shrinking } \\
\hline & 10 & 20 & 40 & 100 & 200 \\
\hline Recent & 0.054 & 0.054 & 0.054 & 0.054 & 0.054 \\
\hline Used & 0.054 & 0.054 & 0.054 & 0.054 & 0.054 \\
\hline Bound & 0.053 & 0.053 & 0.053 & 0.053 & 0.053 \\
\hline
\end{tabular}

Table 10: $\mathrm{C}=100$, caching space $=?(\mathrm{Mb})$ 
Experiments with large caching space show that the caching strategy is very important to save time. If the caching space is sufficient enough, the Rate $_{k e}$ will be very low, leading to great reduction of kernel evaluation. For example, Bound achieves 0.028 of Rate Re $_{\text {in }} w 8 a$ data set of table 10 , when no shrinking is used and caching space is set 200 .

Usps data set is a multi-class problem. All three strategies on this data set show good similar performance(the Rate $_{k e}$ is very low, around 0.05). There are two main explanations leading to such an outcome, one is that the software $L I B S V M$ decomposes a multi-class problem into pairwise binary classification problems. The size of training set becomes small. The other is that the size of each class is small. Therefore, even if the caching space is small, it is enough for those one-againstone binary classifications.

In Table 10, all results show that Bound perform best in all settings. In Table 9, Bound perform best in most settings, but we also note several results of Recent are better than Bound. A reasonable explanation is that the data sets are different because of the randomness produced in the process cross validation.

Overall, all results in Table 9 and Table 10 are further less than 1, which demonstrate that the caching strategies are effective to reduce the computational complexity. Probabilities of examples being selected in working set mainly contribute to the efficiency of caching. By considering probabilities of examples being selected in working set, our caching strategy achieve best performance on most of data sets.

\section{Conclusions}

Working-set selection, caching and shrinking are three components which are related to the computational complexity of the training SVMs. Previous works mainly focused on the working-set selection, while only a little works focused on the caching and shrinking strategies. In this paper, we propose an improved caching strategy, taking into account probabilities of samples being selected into working set in the training of SVMs. Experimental results on several benchmark data sets show the performance of our strategy is better than existing ones used in $L I B S V M$ and $S V M^{\text {light }}$.

In the current stage we mainly deal with caching strategy. Good combination of decomposition algorithms, shrinking and caching strategies may further improve the efficiency of training
SVMs. Our further work will focus on this combination.

\section{References}

[1] V. Vapnik. The Nature of Statistical Learning Theory. Springer Verlag, 1995.

[2] I. B.E.Boser and V.N.Vapnik. A training algorithm for optimal margin classifiers. In $D$. Haussler, editor,Proceedings of the 5th Annual ACM Workshop on Computational Learning Theory,ACM Press, pages 144-152, 1992.

[3] Nello Cristianini and John Shawe-Taylor An Introduction to Support Vector Machines and Other Kernel-based Learning Methods, Cambridge University Press ,2000.

[4] R.-E. Fan, P.-H. Chen, and C.-J. Lin. Working set selection using second order information for training SVM. Journal of Machine Learning Research, 6:1889-1918, 2005.

[5] C. I. Tobias Glasmachers. Maximum-gain working set selection for svms. Journal of Machine Learning Research, 7:1437-1466, July 2006.

[6] T. Joachims. 11 in: Making Large-Scale SVM Learning Practical.Advances in Kernel Methods - Support Vector Learning. B. Schölkopf and C. Burges and A. Smola (ed.), MIT Press, 1999.

[7] R. F. E. Osuna and F. Girosi. Improved training algorithm for support vector machines. In $J$. Principe, L. Giles, N. Morgan, and E. Wilson, editors, Neural Networks for Signal Processing,IEEE Press,, VII:276-285, 1997.

[8] J. Platt. Fast training of support vector machines using sequential minimal optimization. In B. Schölkopf, C. J. C.Burges, and A. J. Smola,editors, Advances in Kernel Methods - Support Vector Learning, chapter 12, pages 185-208. MIT Press, 1999.

[9] S. S. Keerthi, S. K. Shevade, C. Bhattacharyya, and K. R. K. Murthy. Improvements to Platt's SMO algorithm for SVM classifier design. Neural Computation, 13:637-649, 2001.

[10] C.-C. Chang and C.-J. Lin. LIBSVM: a library for support vector machines, 2001. Software available at http://www.csie.ntu.edu.tw/ cjlin/libsvm.

[11] P.-H. Chen, R.-E. Fan, and C.-J. Lin. A study on SMO-type decomposition methods for support vector machines. IEEE Transactions on Neural Networks, 17:893-908, July 2006. 